\title{
Hypersomnia following uvulopalatopharyngoplasty for snoring
}

\author{
R.P. Smith*, J-L. Pépin*, J.R. Catterall*, P.A. Lévy ${ }^{\#}$
}

\begin{abstract}
Hypersomnia following uvulopalatopharyngoplasty for snoring. R.P. Smith, J-L. Pépin, J.R. Catterall, P.A. Lévy. (C) ERS Journals Ltd 1999.

ABSTRACT: This report describes three cases who underwent uvulopalatopharyngoplasty for severe snoring and who subsequently developed progressive excessive daytime sleepiness. All three cases were shown to have sleep fragmentation as a result of non-apnoeic episodic upper airway narrowing. These cases raise the possibility that increased upper airway resistance during sleep may be exacerbated or even caused by uvulopalatopharyngoplasty. Ideally, sleep-disordered breathing should be carefully excluded before this surgery is offered as treatment for severe snoring.

Eur Respir J 1999; 14: 239-241.
\end{abstract}

Keywords: Excessive daytime sleepiness oesophageal manometry snoring upper airway resistance syndrome uvulopalatopharyngoplasty

Received: May 181998 Accepted after revision February 21999

Supported by grants from Region RhôneAlpes (Hypoxie), Royal College of Physicians (Edinburgh), and Bristol Royal Infirmary Respiratory Department.
Uvulopalatopharyngoplasty (UPPP) is increasingly being used to treat severe snoring that is a social nuisance when conservative measures such as reduction in weight and alcohol consumption have failed. There are a number of well documented complications [1, 2], but in careful hands these are regarded as uncommon. This report describes three cases who underwent UPPP for severe snoring and who subsequently presented with disabling hypersomnia arising from sleep fragmentation caused by nonapnoeic episodic upper airway narrowing.

\section{Case report}

A 43-yr-old male presented with hypersomnia, difficulty concentrating and poor memory. He had a long history of severe snoring for which he had undergone UPPP 6 months previously. Prior to surgery he had complained of a degree of daytime sleepiness with an Epworth Sleepiness Scale (ESS) score of 12/24 [3], but polysomnography had not revealed a significant level of sleep-disturbed breathing (apnoea/ hypopnoea index (AHI) 3.36 events $\cdot h^{-1}$ ). Following surgery there was no improvement in his snoring and his hypersomnia became much worse, such that he was having difficulty performing his clerical job in a tax office. He was not overweight at $64 \mathrm{~kg}$ (body mass index (BMI) $20.8 \mathrm{~kg} \cdot \mathrm{m}^{-2}$ ), which had not changed since his operation. Standard polysomnography, which included oesophageal manometry to measure respiratory effort, was repeated at this stage and demonstrated normal oximetry with an AHI of only 4.8 events $\cdot \mathrm{h}^{-1}$. Recurrent episodes of upper airway resistance (UAR) (UAR index 12.5 events $\cdot \mathrm{h}^{-1}$ ) were demonstrated with negative pleural pressure swings of up to $27 \mathrm{cmH}_{2} \mathrm{O}$ (negative pleural pressure swings of $>12 \mathrm{cmH}_{2} \mathrm{O}$ during sleep are considered abnormal) indicating an UAR syndrome. He refused continuous positive airway pressure (CPAP) therapy and has been referred for mandibular advancement surgery (genioglossal advancement and hyoid fixation to the superior border of the thyroid cartilage).

\section{Case 2}

A 43-yr-old male presented with disabling excessive daytime sleepiness. He had a long history of socially embarrassing snoring and had undergone UPPP 7 yrs previously. Prior to surgery he had reported unrefreshing sleep and a degree of daytime hypersomnia which had not been quantified. Pre-operative polysomnography had demonstrated normal nocturnal oximetry but he did show evidence of mild sleep-disturbed breathing at this stage with an AHI of 10 events $\cdot h^{-1}$ (predominantly hypopnoeas). The UPPP did not help his snoring and over subsequent years his daytime hypersomnia worsened. He was slightly overweight at $93 \mathrm{~kg}$ (his weight prior to surgery had been $90 \mathrm{~kg}$ ) and he had a BMI of $27.5 \mathrm{~kg} \cdot \mathrm{m}^{-2}$. His ESS score was markedly elevated at $18 / 24$ and polysomnography was repeated using oesophageal manometry to measure respiratory effort. This showed a moderate number of oxygen desaturation dips with an AHI of 24.8 events $\cdot h^{-1}$ (almost all hypopnoeas) which were associated with large negative pleural pressure swings of up to $40 \mathrm{cmH}_{2} \mathrm{O}$. This patient, therefore, had an obstructive hypopnoea syndrome associated with significant episodes. He was successfully instigated on nocturnal nasal CPAP therapy with a resolution of his daytime sleepiness.

\section{Case 3}

A 48-yr-old male presented with worsening daytime sleepiness. He had been a heavy snorer without excessive daytime sleepiness for $10 \mathrm{yrs}$ and had undergone UPPP 5 yrs previously which had been unsuccessful, resulting in a recurrence of his snoring within several months. A polysomnography had not been performed pre-operatively. Subsequently, he noticed increasing daytime sleepiness with poor memory. His weight was $71 \mathrm{~kg}$ and his BMI was normal at $24.2 \mathrm{~kg} \cdot \mathrm{m}^{-2}$, but his ESS score was significantly elevated at 17/24. Standard polysomnography, including oesophageal manometry to measure respiratory effort, demonstrated an AHI of 14.9 events $\cdot h^{-1}$ which was comprised almost entirely of

\#Dept of Respiratory Medicine, Sleep Laboratory and PRETA Laboratory, CHRU, Grenoble, France. *Respiratory Dept, Bristol Royal Infirmary, Bristol, UK. Correspondence: P.A. Lévy, EFCR, Pneumologie, Rez-de-chausée-haut, CHRU, 38043, Grenoble Cedex 9, France. Fax: 33476765617. 
hypopnoeas. In addition he had a moderate number of episodes (UAR index 12.2 events $\cdot \mathrm{h}^{-1}$ ) which were associated with pleural pressure swings of up to $37 \mathrm{cmH}_{2} \mathrm{O}$. This patient, therefore, had an obstructive hypopnoea syndrome associated with significant UAR. Nocturnal nasal CPAP therapy corrected his sleep-disturbed breathing and produced a dramatic improvement in his daytime symptoms which was maintained at six month follow-up.

All three patients were shown to possess a narrowing of the oropharynx due to a thickening of the residual soft palate when the upper airway was imaged with cephalometry and pharyngeal computerized tomography following UPPP (fig. 1). Although none had oesophageal pressure monitoring as part of the pre-operative assessment, they were all shown to have significant numbers of upper airway nonapnoeic obstructive episodes associated with large pleural pressure swings and cortical arousals on elecroencephalography (EEG) recordings following their surgery.

\section{Discussion}

The distress and embarrassment caused by snoring can be considerable. UPPP is one of a number of procedures increasinglybeingused toalleviate this social problemifothermeasures. such as reduction in weight or alcohol intake have failed.
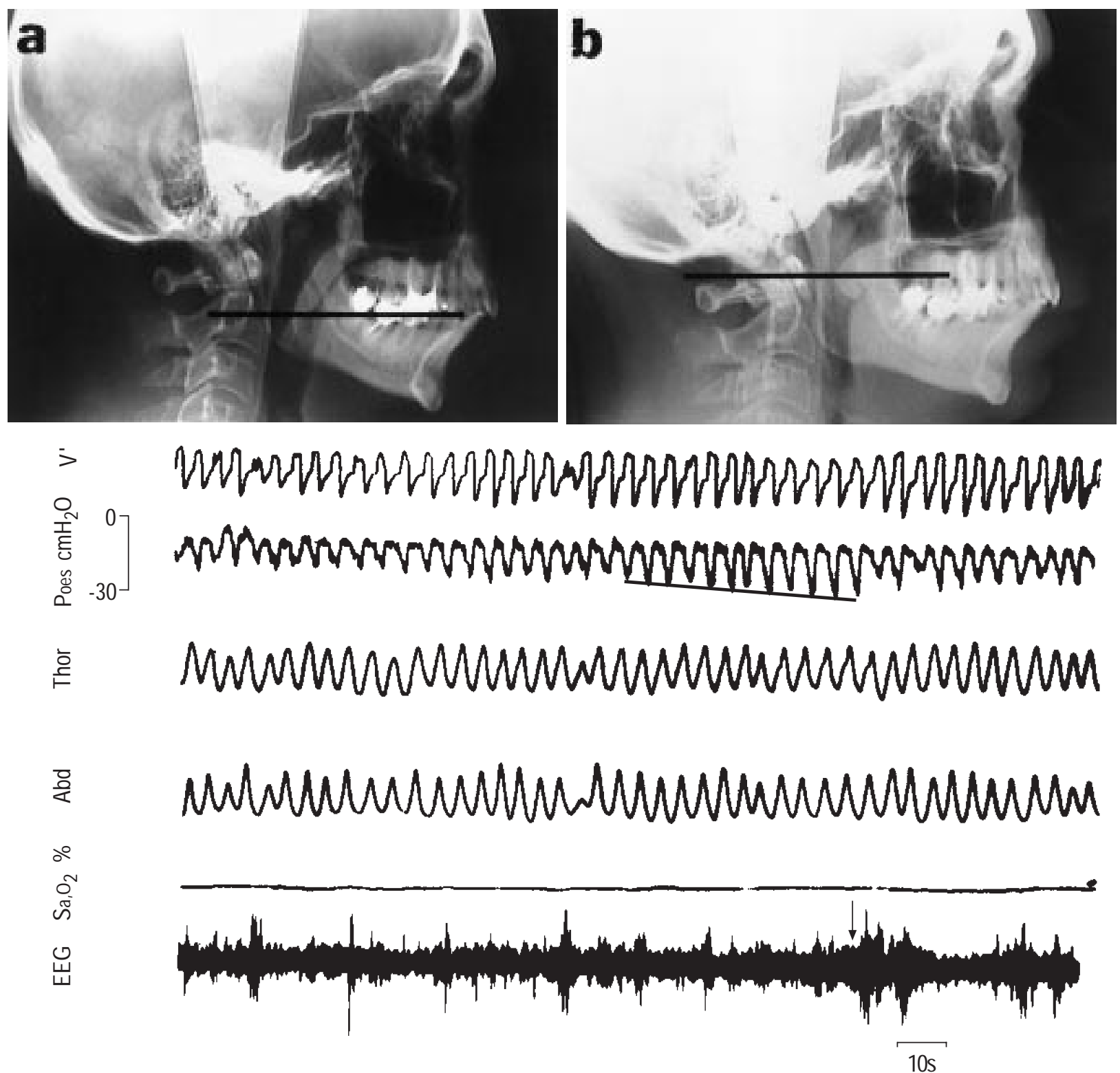

Fig. 1. - Cephalometry of case 1: a) before; and b) after uvulopalatopharyngoplasty. The solid black lines indicate the narrowest point of the oropharynx, identified by concurrent computerized tomography scans (not shown). Marked narrowing of the airway lumen caused by protrusion of the residual soft palate can be clearly seen in the post-operative film. c) Polysomnography trace showing airflow $\left(V^{\prime}\right)$ oesophageal pressure (Poes), thoracic movement (Thor), abdominal movement (Abd), oxygen saturation $\left(\mathrm{S}_{\mathrm{a}}, \mathrm{O}_{2}\right)$ and electroencephalography (EEG), measured using a nasal mask pneumotachograph. The bucal thermistor (not shown) did not show any evidence of oral airflow during this particular period. Here, an episode of upper airway resistance is demonstrated by the progressive rise in negative oesophageal pressure (representing respiratory effort, solid black line) in the absence of diminished airflow, abnormal chest and abdominal wall movements and hypoxaemia. This upper airway resistance is reversed when arousal from sleep occurs (indicated by arrow on EEG trace). Arousal restores upper airway muscle tone, which reduces the resistance to airflow, thus allowing the resumption of normal breathing (oesophageal pressure returns to normal). 
However, the operation is not without complications [1, 2], and its efficacy, even in carefully selected patients, is variable.

Sleep-disordered breathing, which may accompany snoring, is common and is estimated to affect $9 \%$ and $4 \%$ of middleaged males and females, respectively [4]. Only a proportion of these will have classical full-blown obstructive sleep apnoea and it is increasingly being recognized that there is another subgroup of patients who have episodic partial upper airway obstruction that does not cause a significant reduction in airflow, but is severe enough to cause large pleural pressure swings and recurrent arousal from sleep. This condition, called the UAR syndrome, tends not to produce complete apnoeas and recurrent hypoxaemia, but the sleep fragmentation arising from the increased respiratory effort can result in disabling hypersomnia and, as with obstructive sleep apnoea, the symptoms can be prevented with nasal CPAP therapy [5, 6]. It is likely that in such patients, the upper airway wall is stiffer than in apnoeic patients and therefore, despite high resistance and large negative inspiratory pressures, airway collapse does not occur and airflow is largely preserved. The absence of hypoxaemia and complete apnoeas makes this condition more difficult to detect using standard sleep laboratory techniques and a sensitive measure of respiratory effort, such as oesophageal pressure monitoring, is often required to detect these nonapnoeic events.

Oesophageal pressure monitoring will not differentiate between an UAR episode, in which airflow is preserved, and a hypopnoea, in which there is a reduction of airflow by at least $30 \%$. For this, a quantitative measure of airflow, such as pneumotachography, is needed, but this is not widely available in clinical practice. Thermally sensitive devices such as thermistors are more commonly used to detect reductions in airflow, but these are only semi-quantitative [7] and subtle changes may be missed. Thermistors were used in cases 1 and 3 in this report and this may have underestimated the number of hypopnoeas and overestimated the number of true UAR episodes. However, there is some controversy at present as to whether distinguishing between these nonapnoeic obstructive events is clinically important. If the final common pathway of microarousal, sleep fragmentation and autonomic activation is the same for both these types of obstructive respiratory episodes, and if both can be reversed with CPAP therapy, then making the distinction using subtle changes in airflow may not be necessary.

The efficacy of UPPP as a primary treatment for sleep apnoea itself is limited. The procedure may not prevent obstructive events if the airway collapse is occurring at a number of levels in the oropharynx [8]. It produces limited objective improvement in snoring if obstructive sleep apnoea is present [8], and the operation may make future CPAP therapy difficult by compromising the seal between the soft palate, tongue and palatal arches [9]. The present patients developed worsening hypersomnia following surgery and they were all shown to have sleep fragmentation in association with episodes of high respiratory effort. These obstructive respiratory events were predominantly a mixture of UAR episodes and hypopnoeas with very few apnoeas or episodes of significant arterial desaturation. It is not certain whether this deterioration was a direct consequence of the UPPP itself or whether it merely represented the natural progression of preexisting sleep-disordered breathing that has been described in mild sleep apnoea [10], but what is clear is that the patients did not benefit from surgery. Although they did not have significant nocturnal apnoeas or oxygen desaturations before surgery, the presence of a degree of hypersomnia and a moderate numbers of hypopnoeas pre-operatively in cases 1 and 2 suggest that a problem may have existed before UPPP.
On the other hand, it has been shown that this operation can cause a thickening of the residual soft palate with protrusion into the pharyngeal airway causing narrowing of the lumen [11]. This was indeed demonstrated by upper airway imaging in each of the present patients, and it one can be postulated that lumenal narrowing and increased stiffness of the pharyngeal wall caused by postoperative scarring combined to produce increased UAR during sleep with consequent sleep fragmentation. Although it is difficult to prove a causal relationship with the UPPP in these cases as oesophageal manometry was not performed pre-operatively, the subsequent worsening of hypersomnia does suggest that the surgery may, at least, have contributed to their increased UAR.

In summary, these case reports illustrate that predicting the outcome of uvulopalatopharyngoplasty can be difficult and they pose the question of whether, in some subjects, this surgery may exacerbate, or even cause, increased upper airway resistance during sleep. A prospective study providing systematic clinical and physiological outcome data for uvulopalatopharyngoplasty is needed. Ideally, sleep disordered breathing should be carefully excluded before such an operation is offered as treatment for severe snoring. Upper airway resistance should be considered in any patient presenting with unexplained excessive daytime sleepiness who does not have detectable apnoeas or hypopnoeas and such cases should be offered oesophageal pressure monitoring or, if this is not available, a trial of nasal continuous positive airway therapy.

\section{References}

1. Croft CB, Golding-Wood DG. Uses and complications of uvulopalatopharyngoplasty. J Laryngol Otol 1990; 104: 871-875.

2. Haavisto L, Suonpää J. Complications of uvulopalatopharyngoplasty. Clin Otolaryngol 1994; 19: 243-247.

3. Johns MW. A new method for measuring daytime sleepiness: the Epworth sleepiness scale. Sleep 1991; 14: 540545.

4. Young T, Palta M, Dempsey J, Skatrud, Weber S, Badr S. The occurrence of sleep disordered breathing among middle-aged adults. N Engl J Med 1993; 328: 1230-1235.

5. Guilleminault C, Stoohs R, Clerk A, Cetel M, Maistros P. A cause of excessive sleepiness: the upper airway resistance syndrome. Chest 1993; 104: 781-787.

6. Strollo PJ, Rogers RM. Current concepts: obstructive sleep apnoea. N Engl J Med 1996; 334: 99-104.

7. Farré R, Montserrat JM, Rotger M, Ballester E, Navajas D. Accuracy of thermistors and thermocouples as flowmeasuring devices for detecting hypopnoeas. Eur Respir $J$ 1998; 11: 179-182.

8. Sher AEK, Schechtman KB, Piccirillo JF. The effficacy of surgical modifications of the upper airway in adults with obstructive sleep apnoea syndrome. Sleep 1996; 19: 156177.

9. Mortimore IL, Bradley PA, Murray JAM, Douglas NJ. Uvulopalatopharyngoplasty may compromise nasal CPAP therapy in sleep apnoea syndrome. Am J Respir Crit Care Med 1996; 154: 1759-1762.

10. Pendlebury ST, Pépin J-L, Veale D, Lévy P. Natural evolution of moderate sleep apnoea syndrome: significant progression over a mean of 17 months. Thorax 1997; 52: 872-878.

11. Langin T, Pépin JL, Pendlebury S, et al. Upper airway changes in snorers and moderate sleep apnoea sufferers after uvulopalatopharyngoplasty (UPPP). Chest 1998; 113: $1595-1603$. 